\title{
Scaling in high-temperature superconductors
}

\author{
by \\ Ian D Lawrie L $^{\dagger}$ \\ Department of Physics, The University of Leeds, Leeds LS2 9JT, UK
}

(March 1994)

\begin{abstract}
A Hartree approximation is used to study the interplay of two kinds of scaling which arise in high-temperature superconductors, namely critical-point scaling and that due to the confinement of electron pairs to their lowest Landau level in the presence of an applied magnetic field. In the neighbourhood of the zero-field critical point, thermodynamic functions scale with the scaling variable $\left(T-T_{c 2}(B)\right) / B^{1 / 2 \nu}$, which differs from the variable $\left(T-T_{c}(0)\right) / B^{1 / 2 \nu}$ suggested by the gaussian approximation. Lowest-Landau-level (LLL) scaling occurs in a region of high field surrounding the upper critical field line but not in the vicinity of the zero-field transition. For $\mathrm{YBaCuO}$ in particular, a field of at least $10 \mathrm{~T}$ is needed to observe LLL scaling. These results are consistent with a range of recent experimental measurements of the magnetization, transport properties and, especially, the specific heat of high- $T_{c}$ materials.
\end{abstract}

PACS numbers: 05.70.Jk 64.60.Fr 74.20.-z 74.30.Ek

${ }^{\dagger}$ e-mail address: i.d.lawrie@uk.ac.leeds 


\section{Introduction}

The superconducting transition in conventional low- $T_{c}$ materials is well described by the Ginzburg-Landau mean field theory. Principally because of the large correlation volume in these materials, the region in which critical fluctuations might be important is too small to be accessible experimentally. In high-temperature superconductors, by contrast, the critical region may be much larger. Widely varying theoretical estimates of the size of this region are obtained according to details of the criterion employed ${ }^{1}$, but marked deviations from mean-field behaviour have been observed $^{2-6}$ over a temperature range of the order of $10 \mathrm{~K}$ above and below $T_{c}$.

Theoretical expectations of the kind of critical behaviour which might be observed are somewhat confused. If fluctuations in the magnetic vector potential can be ignored, then the zero-field transition ought to be a critical point in the universality class of the 3-dimensional XY model, as is the superfluid transition in ${ }^{4}$ He. When magnetic fluctuations are included, a renormalization-group analysis by Halperin, Lubensky and $\mathrm{Ma}^{7}$ for a $(4-\epsilon)$-dimensional system reveals a runaway of renormalization-group trajectories, which these authors interpreted as a signal of a weakly first-order transition. This interpretation is confirmed by an explicit construction of the free energy ${ }^{8}$. On the other hand, a renormalization-group analysis in $(2+\epsilon)$ dimensions $^{9}$ indicates a second-order transition in the universality class of the $C P^{N-1}$ model in the limit $N \rightarrow 1$, while a lattice simulation of Dasgupta and Halperin ${ }^{10}$ is consistent with inverted XY critical behaviour. In high- $T_{c}$ cuprates, the region in which a first-order transition or inverted XY behaviour might be detected is probably extremely small. These are strongly type-II materials with penetration

depths in excess of $1000 \AA$. In this situation, renormalization-group trajectories pass 
very close to the ordinary XY fixed point, suggesting that magnetic fluctuations can indeed be ignored except in a very narrow range of temperatures near $T_{c}$.

Experience of critical phenomena in, for example, fluids and magnets suggests that, in the presence of an applied magnetic field $B$, there should be a critical region in which thermodynamic quantities assume the scaling form $A(T, B)=B^{\alpha_{A}} \mathcal{A}(x)$, where $\alpha_{A}$ is a critical exponent associated with the quantity $A$ and the scaling variable is an appropriate ratio of scaling fields. In principle, the correct scaling fields would emerge from a renormalization-group analysis, but this analysis is very difficult in the presence of an applied field. An early calculation of Prange ${ }^{11}$ using the gaussian approximation suggests that $B$ occurs in the combination $B \xi^{2}$, where $\xi$ is the zero-field coherence length, so that the scaling variable should be $x=$ $\left(T-T_{c}\right) / B^{1 / 2 \nu}$, where $\nu$ is the coherence-length exponent. We shall argue that this is not quite correct, however, and that the scaling variable should be $x=$ $\left(T-T_{c 2}(B)\right) / B^{1 / 2 \nu}$, where the line $T=T_{c 2}(B)$ is a renormalized version of the line usually denoted by $H_{c 2}(T)$, the upper critical field in mean field theory. In the gaussian approximation, with $\nu=\frac{1}{2}$, these two scaling variables differ only by an additive constant.

A different scaling form for thermodynamic functions (which is not directly associated with a phase transition) arises in the lowest Landau level (LLL) approximation $^{12-14}$, which is usually thought to be valid in the neighbourhood of the $H_{c 2}(T)$ line. Here, the appropriate scaling variable is $y=\left(T-T_{c 2}(B)\right) / B^{\phi}$, where the exponent $\phi$ has the value $\phi=\frac{2}{3}$ in 3 dimensions or $\phi=\frac{1}{2}$ in 2 dimensions. This scaling behaviour is well verified experimentally for conventional superconductors ${ }^{12-16}$, but the type of scaling which applies to high- $T_{c}$ materials is at present a matter of some 
controversy. It has been claimed by Welp et $a l^{17}$ that magnetization, conductivity and specific heat data for $\mathrm{YBaCuO}$ are consistent with LLL scaling. On the other hand, Inderhees et $a l^{4}$ and Salamon et $a l^{5}$ claim that experimental data is more nearly consistent with critical-point scaling. In fact, the predicted scaling forms for the magnetization and conductivity are probably too similar to be distinguished experimentally. For the specific heat, the LLL scaling fit exhibited by Welp et al is rather poor, and is achieved only by the introduction of a prefactor which has no theoretical basis.

In this work, we use a Hartree approximation to study the interplay of criticalpoint and LLL scaling in an isotropic, $d$-dimensional system. Although materials such as $\mathrm{YBaCuO}$ are anisotropic, layered systems, we expect that the results of this study should provide a reasonable qualitative guide to the scaling behaviour in the vicinity of the critical point, where the coherence length is much larger than the interlayer spacing. The Hartree approximation is in any case too crude to give accurate numerical estimates either of critical exponents or of scaling functions. We find that critical scaling may be expected in a region of small fields and temperatures near $T_{c}(0)$, while LLL scaling occurs in a region which surrounds the $H_{c 2}(T)$ line, but stops short of the zero field critical point at a field value which we estimate at between 10 and 100 T. The Hartree approximation is described in section 2 below, and a criterion for the validity of the LLL approximation is obtained in section 3 . Sections 4 - 6 discuss scaling behaviour of the field-dependent coherence length, the specific heat and the electrical conductivity. A comparison with recent experimental measurements is made in section 7 , and our conclusions are summarized in section 8. 


\section{Hartree approximation}

The Ginzburg-Landau-Wilson reduced Hamiltonian may be written in a standard form as

$$
\mathcal{H}=\int d^{d} x\left[|(\nabla-i e \mathbf{A}) \phi|^{2}+t_{0}|\phi|^{2}+\frac{1}{2} \lambda|\phi|^{4}\right]
$$

where, in the critical region, $t_{0}$ can be taken as linear in temperature and $\lambda$ as a constant. We assume throughout that the magnetic flux density $\mathbf{B}=\nabla \times \mathbf{A}$ is uniform, and equal to the applied field. Thus, the vector potential $A$ is not a fluctuating variable, and the expectation value of a quantity $f(\phi)$ is

$$
\langle f(\phi)\rangle=\int \mathcal{D} \phi f(\phi) \exp (-\mathcal{H}) / \int \mathcal{D} \phi \exp (-\mathcal{H})
$$

For a $d$-dimensional system, a convenient gauge choice is $\mathbf{A}=\frac{1}{2} B(-y, x, \mathbf{0})$, where $\mathbf{0}$ denotes the components in the $(\mathrm{d}-2)$ dimensions "parallel" to the magnetic field. We implement an approximation of the Hartree type by introducing an approximate Hamiltonian

$$
\mathcal{H}_{0}=\int d^{d} x\left[|(\nabla-i e \mathbf{A}) \phi|^{2}+\left(t_{0}+\mu\right)|\phi|^{2}\right]
$$

where $\mu$ is determined self-consistently by requiring that $\mathcal{H}$ and $\mathcal{H}_{0}$ have the same expectation value in the ensemble of $\mathcal{H}_{0}$ :

$$
\left\langle\mathcal{H}-\mathcal{H}_{0}\right\rangle_{0}=\frac{\lambda}{2}\left\langle|\phi|^{4}\right\rangle_{0}-\mu\left\langle|\phi|^{2}\right\rangle_{0}=0
$$

The expectation values are easily expressed in terms of a sum over Landau levels as $\left\langle|\phi|^{2}\right\rangle_{0}=I$ and $\left\langle|\phi|^{4}\right\rangle_{0}=2 I^{2}$, where

$$
I=\frac{2 e B}{4 \pi} \sum_{n} \int \frac{d^{d-2} k}{(2 \pi)^{d-2}} \frac{1}{k^{2}+2 e B n+t_{0}+\mu+e B} .
$$

On defining

$$
\tilde{t}=t_{0}+e B+\mu
$$


we obtain the constraint equation (2.4) in the form

$$
\tilde{t}=t_{0}+e B+\frac{\lambda}{4 \pi} 2 e B \sum_{n} \int \frac{d^{d-2} k}{(2 \pi)^{d-2}} \frac{1}{k^{2}+2 e B n+\tilde{t}} .
$$

The sum and integral in this expression is divergent, unless the integration is restricted by an upper cutoff, of the order of an inverse lattice spacing. However, this divergence can be eliminated by an additive renormalization of the temperature. On carrying out the sum and angular integrations, we find

$$
\tilde{t}=t+e B+\hat{\lambda}(2 e B)^{d / 2-1} f(\tilde{t} / 2 e B)
$$

where $\hat{\lambda}=\lambda /(4 \pi)^{d / 2}$, the renormalized temperature variable is $t=t_{0}+\hat{\lambda} \int_{0}^{\infty} d x x^{-d / 2}$ and

$$
f(z)=\int_{0}^{\infty} d x x^{-d / 2}\left[\frac{x e^{-x z}}{1-e^{-x}}-1\right] .
$$

For $2<d<4$, this integral is finite. We are not able to evaluate it analytically, but its limiting behaviour for small and large values of $z=\tilde{t} / 2 e B$ can be obtained straightforwardly. When $\mathrm{z}$ is small, we have

$$
f(z) \approx f_{0} z^{d / 2-2}
$$

where $f_{0}=\int_{0}^{\infty} d x x^{1-d / 2} e^{-x}(=\sqrt{ } \pi$ for $d=3)$. In this limit, the constraint equation reads

$$
\tilde{t} \approx t+e B+\hat{\lambda} f_{0}(2 e B) \tilde{t}^{d / 2-2}
$$

This limit corresponds to the lowest Landau level (LLL) approximation, where the sum in (2.7) is approximated by the term $n=0$. In this approximation, $\tilde{t}$ can be expressed in a scaling form, which is inherited by various thermodynamic functions ${ }^{12}$, namely

$$
\tilde{t} \approx\left(\hat{\lambda} f_{0} e B\right)^{\phi} \tau_{L L L}(y)
$$


where $\phi=2 /(6-d)$ (so $\phi=2 / 3$ in 3 dimensions), $y=(t+e B) /\left(\hat{\lambda} f_{0} e B\right)^{\phi}$ and the scaling function $\tau_{L L L}$ is the solution of

$$
\tau_{L L L}(y)=y+2 \tau_{L L L}(y)^{1-1 / \phi}
$$

In the opposite limit, $z \rightarrow \infty$, corresponding to very low fields, we have

$$
f(z) \approx-f_{\infty} z^{d / 2-1}
$$

where $f_{\infty}=\int_{0}^{\infty} d x x^{-d / 2}\left(1-e^{-x}\right)(=2 \sqrt{ } \pi$ for $d=3)$.

\section{Validity of the lowest Landau level approximation}

One can, of course, make the approximation of neglecting all Landau levels except the lowest without also invoking the Hartree approximation, and perturbative calculations based on such an approximation have been pursued by several authors ${ }^{14,15,18}$. One finds that the scaling form (2.12) persists, though the scaling function is no longer that given by solving (2.13). It is clearly essential to know where in the phase diagram the LLL approximation is likely to be valid, and we address this question within the Hartree approximation in the following way. We suppose that the function $f(z)$ is well approximated by (2.10) whenever $z$ is smaller than some fiducial value, say $\epsilon$. For $z=\tilde{t} / 2 e B=\epsilon$, the constraint (2.11) reads

$$
t=-e B\left[1-2 \epsilon+2 \hat{\lambda} f_{0}(2 e B \epsilon)^{-\psi}\right]
$$

where $\psi=\phi^{-1}-1=2-d / 2(\psi=1 / 2$ in three dimensions $)$ and defines a locus in the $(t, B)$ plane, which is shown schematically in figure 1. Points for which $z<\epsilon$ lie below this line and this, therefore, is the region in which we might expect the LLL approximation to be valid. However, our analysis is based on the assumption that the order parameter $\langle\phi\rangle$ is negligibly small. This assumption presumably becomes 
invalid at some distance below the mean-field $H_{c 2}(T)$ line $t=-e B$, but we are unable to determine whether some form of the LLL approximation survives with $\langle\phi\rangle \neq 0$. Supposing that the order parameter is indeed negligible above the line labelled $\langle\phi\rangle \sim 0$ in figure 1 (whose location we cannot determine precisely), we are led to the conservative conclusion that the LLL approximation should be valid in the roughly wedge-shaped region labelled LLL in the figure. This region encloses a highfield, low-temperature portion of the line $t=-e B$, but stops short of the critical point $t=B=0$. As the condition for the accuracy of the LLL approximation is made more stringent, by reducing the value of $\epsilon$, the wedge recedes to higher fields and lower temperatures.

\section{Scaling of the coherence length}

From the appearance of $\tilde{t}$ in the propagator in (2.7), we can identify this quantity in terms of a temperature- and field-dependent coherence length $\xi(t, B)$ as $\tilde{t}=$ $\xi(t, B)^{-2}$. We find from the constraint equation (2.8) that it can be expressed in a two-parameter scaling form as

$$
\tilde{t}=(2 e B) \tau_{B}(\theta, \delta)
$$

where the scaling variables are

$$
\begin{gathered}
\theta=\hat{\lambda}^{-1}(t+e B)(2 e B)^{-1 / 2 \nu}, \\
\delta=\hat{\lambda}^{-1}(2 e B)^{\omega / 2},
\end{gathered}
$$

with exponents $\nu=1 /(d-2)$ and $\omega=4-d$, and the scaling function $\tau_{B}$ is the solution of

$$
\delta \tau_{B}=\theta+f\left(\tau_{B}\right)
$$


It is straightforward to show that $\tau_{B}$ has a double power series expansion in $\theta$ and $\delta$. For small fields and temperatures close to $T_{c}$, we can take the limit $\delta \rightarrow 0$ with $\theta$ fixed, to obtain the one-parameter scaling form

$$
\tilde{t} \approx(2 e B) \tau_{B}(\theta, 0)=(2 e B) f^{-1}(-\theta) .
$$

This should be valid in a region near the critical point $t=B=0$, indicated schematically by the shaded "Critical" region in figure 1. According to (2.10) and (2.14), the critical-point scaling function $\tau_{B}(\theta, 0)=f^{-1}(-\theta)$ has the limiting forms

$$
\begin{gathered}
\tau_{B}(\theta, 0) \approx\left(\frac{\theta}{f_{\infty}}\right)^{2 /(d-2)}, \quad \theta \rightarrow+\infty, \\
\tau_{B}(\theta, 0) \approx\left(-\frac{\theta}{f_{0}}\right)^{-2 /(4-d)}, \quad \theta \rightarrow-\infty .
\end{gathered}
$$

In general, the regions where the LLL scaling form (2.12) and the critical scaling form (4.5) hold must be distinct. There may, however, be a crossover region in which both forms are approximately valid. This requires

$$
\tau_{B}(\theta, 0) \sim \theta^{-\sigma}
$$

and

$$
\tau_{L L L}(y) \sim y^{-\sigma},
$$

where $\sigma=2 \nu(1-\phi) /(2 \nu \phi-1)$. In the Hartree approximation, we have $\sigma=2 /(4-d)$ and we find from (2.13) and (4.7) that there is such a crossover region, namely the region of very low field below $T_{c}$, where $\theta$ and $y$ are both large and negative. It is, however, in this region that the approximation $\langle\phi\rangle \approx 0$ is likely to fail, so (4.8) and (4.9) are not necessarily meaningful in this region. Moreover, we do not know whether these limiting forms of the scaling functions are valid beyond the Hartree 
approximation, so it is not clear whether the crossover region would be accessible in real materials.

In the usual way, the scaling relation (4.1) can be reformulated as

$$
\tilde{t}=|t+e B|^{2 \nu} \tau_{t}^{ \pm}(\beta, \eta)
$$

where $\beta=2 e B|t+e B|^{-2 \nu}, \eta=|t+e B|^{\omega \nu}$ and the + and - branches of the scaling function refer to $t>-e B$ and $t<-e B$. It is straightforward to show that $\tau_{t}^{ \pm}$has a double power series expansion in $\beta$ and $\eta$. The form (4.1) is, however generally the more convenient.

\section{Scaling of the specific heat}

Identification of the specific heat within the Hartree approximation is somewhat ambiguous. On the one hand, we can define a Hartree approximation to the free energy density, $F_{0}=-V^{-1} \ln \left[\int \mathcal{D} \phi \exp \left(-\mathcal{H}_{0}\right)\right]$, where $\mathcal{H}_{0}$ is the approximate Hamiltonian introduced in (2.3), and identify $C=\partial^{2} F_{0} / \partial t^{2}$. On the other hand, we can identify the entropy density of the original Ginzburg-Landau-Wilson model (2.1) as $S=-\frac{1}{2}\left\langle|\phi|^{2}\right\rangle$ and the specific heat as $C=\partial S / \partial t$, and evaluate this quantity in the Hartree approximation:

$$
C=-\frac{1}{2} \frac{\partial}{\partial t}\left\langle|\phi|^{2}\right\rangle_{0}
$$

These two definitions are not equivalent. The latter definition was adopted, for example, by Bray ${ }^{12}$ and is the one we use here. It is slightly simpler, and agrees with the natural definition of the specific heat in the many-component limit ${ }^{19}$, which is largely equivalent to the Hartree approximation. Up to a non-universal constant 
prefactor, the singular part of the specific heat is then given by

$$
C=1-\frac{\partial \tilde{t}}{\partial t}
$$

Scaling behaviour of the specific heat now follows directly from that of $\tilde{t}$. In the lowest Landau level approximation, we have

$$
C \approx 1-\tau_{L L L}^{\prime}(y)
$$

with the scaling variable $y$ and scaling function $\tau_{L L L}$ defined as in $(2.12)-(2.13)$, and this naturally reproduces Bray's result ${ }^{12}$. Corresponding to the two-parameter scaling form (4.1) for $\tilde{t}$, we find

$$
C=1-\hat{\lambda}^{-1}(2 e B)^{-\alpha / 2 \nu} \mathcal{C}(\theta, \delta)
$$

where $\alpha=2-d \nu=-(4-d) /(d-2)$ and

$$
\mathcal{C}(\theta, \delta)=\frac{\partial}{\partial \theta} \tau_{B}(\theta, \delta)
$$

This relation between the scaling functions for the specific heat and the coherence length is a special feature of the Hartree approximation, as are the associated relations

$$
\alpha=1-2 \nu=-\omega \nu
$$

between the specific heat exponent $\alpha$, the coherence length exponent $\nu$ and the correction to scaling exponent $\omega$. It seems plausible, however, that the scaling form (5.4) should be more generally valid. That is, we expect that a region should exist in which both the asymptotic critical behaviour of the specific heat and the leading corrections are described by a function of the form

$$
C=C_{1}-C_{2} B^{-\alpha / 2 \nu} \mathcal{C}\left(\frac{t+B}{B^{1 / 2 \nu}}, u B^{\omega / 2}\right)
$$


In this expression, $C_{1}$ and $C_{2}$ are non-universal amplitudes and $u$ is the scaling field associated with the leading corrections. With $t$ and $B$ appropriately scaled (so that, in particular, the line $H_{c 2}(T)$ becomes $t+B=0$ ), the scaling function $\mathcal{C}$ would be universal.

This scaling form has some familiar consequences. In the limit $B \rightarrow 0$ with $t>0$, we can use (4.6) to find

$$
C \approx c_{1}-c_{2} t^{-\alpha}
$$

with appropriate (non-universal) constants $c_{1}$ and $c_{2}$. Since $\alpha$ is negative, the specific heat rises to a cusp at the critical point $t=0$. Below $T_{c}$ in zero field, a real superconductor is presumably in its Meissner phase, with $\langle\phi\rangle \neq 0$, where our approximations are not valid. The field dependence at $T=T_{c}$ or $t=0$ is given by

$$
C=C_{1}-C_{2} B^{-\alpha / 2 \nu} \mathcal{C}\left(B^{1-1 / 2 \nu}, u B^{\omega / 2}\right)
$$

For small fields, we have the asymptotic power law behaviour $C \approx c_{1}-c_{2}^{\prime} B^{-\alpha / 2 \nu}$. Corrections to this asymptotic behaviour now involve both $B^{1-1 / 2 \nu}$ and $B^{\omega / 2}$. In the Hartree approximation, it happens that $1-1 / 2 \nu=\omega / 2=(4-d) / 2(=1 / 2$ in three dimensions). A real superconductor, however, is probably characterized by the exponents of the three-dimensional XY model, for which $\omega / 2 \approx 0.4$ and $1-1 / 2 \nu \approx 0.25$, and the leading correction would be that involving $B^{1-1 / 2 \nu}$.

Of rather greater interest is the behaviour near the line $t+e B=0$, corresponding to the mean-field $H_{c 2}(T)$ line. According to our earlier discussion, one should eventually pass from the critical region into a region where the lowest Landau level approximation becomes good. Within the Hartree approximation, the two-parameter scaling form (5.4) is exact, and reduces to the LLL scaling form (5.3) in the limit that 
$\delta \sim B^{\omega / 2}$ is large. In general, corrections to the asymptotic, one-parameter critical scaling may have many contributions beyond those associated with the scaling field $u$ indicated in (5.7), and all of these might be important in the the region between the critical and LLL regimes. It is interesting to speculate, on the other hand, that this may not be so, and that a crossover to LLL behaviour can be described by the two-parameter scaling function $\mathcal{C}(\theta, \delta)$. This requires that, when $\delta$ is large,

$$
\mathcal{C}(\theta, \delta) \approx \delta^{\alpha / \omega \nu} \mathcal{C}_{L L L}\left(\theta \delta^{-(2 \nu \phi-1) / \omega \nu}\right)
$$

where the argument $\theta \delta^{-(2 \nu \phi-1) / \omega \nu}$ coincides, up to a constant, with the argument $y=(t+e B) /\left(\hat{\lambda} f_{0} e B\right)^{\phi}$ appearing in (5.3). We do, of course, find such behaviour in the Hartree approximation.

Unfortunately, mapping out a two-parameter scaling function experimentally to test the behaviour suggested in (5.10) would be extremely difficult. Theoretically, it is important to note that the crossover mechanism exemplified by (5.10) is quite different from that expected at a multicritical point. In the mean field theory of type II superconductors, the amplitude of the order parameter in the Abrikosov vortex lattice vanishes continuously at the line $H_{c 2}(T)$, and this is sometimes described as a second-order phase transition. It is possible to speculate that the specific heat, for example, should exhibit an anomaly along this line, perhaps governed by a critical exponent $\alpha^{\prime}$. In that case, the transition at $(T, B)=\left(T_{c}, 0\right)$ would be a multicritical point, and one would expect the one-parameter scaling function to incorporate the anomaly:

$$
\mathcal{C}(\theta, 0) \sim|\theta|^{-\alpha^{\prime}}
$$

as $\theta \rightarrow 0$. If such an anomaly exists, it is too weak to be resolved by any experiment known to us. 


\section{Scaling of the conductivity}

Electrical transport properties of a superconductor can be investigated by using a time-dependent Ginzburg-Landau equation to describe the dynamics. A method of calculation is described in detail by Ullah and Dorsey ${ }^{20}$ who use a Hartree approximation to study scaling behaviour in the LLL regime. Using essentially the same method, we have calculated the conductivities $\sigma_{\|}$and $\sigma_{\perp}$ corresponding to a current parallel or transverse to the applied magnetic field. We find that both conductivities can be written in the two-parameter scaling form

$$
\sigma_{a}=B^{-(2+z-d) / 2} \mathcal{S}_{a}(\theta, \delta)
$$

with a dynamical exponent $z=2$, but with different scaling functions $\mathcal{S}_{\|}$and $\mathcal{S}_{\perp}$. In the limit of large $\delta$, the LLL scaling properties of the two conductivities are different, however, and we find

$$
\sigma_{\|} \approx \mathcal{S}_{L L L, \|}(y)
$$

where $\mathcal{S}_{L L L, \|}=$ const $\times \tau_{L L L}(y)^{-1 / \phi}$, while

$$
\sigma_{\perp} \approx B^{-(z+2-d) \phi / 2} \mathcal{S}_{L L L, \perp}(y)
$$

with $\mathcal{S}_{L L L, \perp}=$ const $\times \tau_{L L L}(y)^{-(z+2-d) / 2}$. The results (6.2) and (6.3) agree with those quoted by Ullah and Dorsey for $d=3$ and $d=2$, except that their 2-dimensional result for $\sigma_{\|}$is quite different from (6.2), having the form $\sigma_{\|} \approx B^{-1 / 2} \mathcal{S}_{L L L, \|}(y)$. We are unable to account for this discrepancy in detail. No doubt, however, it has to do with the fact that, whereas we have considered an isotropic $d$-dimensional material, Ullah and Dorsey deal with a layered 3-dimensional system, and obtain a two-dimensional limit by taking a large interlayer spacing. 


\section{Comparison with experiment}

While the Hartree approximation may well provide a useful guide to the scaling behaviour to be expected in real high- $T_{c}$ materials, it is numerically rather inaccurate, having critical exponents $\alpha=-1$ and $\nu=1$ in three dimensions, compared with $\alpha \approx-0.01$ and $\nu \approx 0.67$ for the 3-dimensional XY model, which might be expected to characterize real materials. Similarly, we do not expect the scaling functions to be numerically accurate, and do not present detailed computations.

Estimates of some gross features of the phase diagram from the Hartree approximation may, however, have some significance. To obtain such estimates, we need to identify the parameters of the model in terms of measurable quantities. We use the customary means of identifying the parameters in (2.1), which is explained, for example, by Tinkham ${ }^{21}$. These must be treated with caution, however, since they are based on the assumption that the entire phase diagram of the superconductor is described by mean field theory, which is not actually the case. In SI units, we find that the constraint equation (2.8) in 3 dimensions takes the form

$$
\tilde{t}=\xi_{0}^{-2}\left(\frac{T}{T_{c}}-1\right)+2 \pi \frac{B}{\Phi_{0}}+4 \pi \frac{\kappa^{2} \mu_{0} k_{B} T_{c}}{\Phi_{0}^{2}}\left(\frac{B}{\Phi_{0}}\right)^{1 / 2} f\left(\frac{\tilde{t} \Phi_{0}}{4 \pi B}\right),
$$

where $\Phi_{0}=2.07 \times 10^{-15} \mathrm{~Wb}$ is the flux quantum, $\mu_{0}$ is the magnetic permeability, which we take to be that of free space $\left(\mu_{0}=4 \pi \times 10^{-7} \mathrm{Hm}^{-1}\right)$ and $\kappa$ is the GinzburgLandau parameter. The quantity $\xi_{0}$ is a characteristic length, of the order of the zero-temperature coherence length.

From the first two terms on the right hand side of (7.1), we find the slope of the $H_{c 2}(T)$ line as

$$
\frac{d B}{d T}=-\frac{\Phi_{0}}{2 \pi \xi_{0}^{2} T_{c}}=-3.29 \times 10^{4}\left(\xi_{0}^{2} T_{c}\right)^{-1}
$$


where $\xi_{0}$ is measured in $\AA$ and $T_{c}$ in $\mathrm{K}$. We can also obtain an estimate for the field strength, denoted by $B_{L L L}$ in figure 1 , at which the line $H_{c 2}(T)$ enters the region of LLL scaling. At this point, the first two terms of (7.1) cancel, and the argument of $f$ is equal to a value $\epsilon$, for which $f(\epsilon) \approx \sqrt{ } \pi \epsilon^{-1 / 2}$. We thus find

$$
B_{L L L}=\frac{\pi\left(\kappa^{2} \mu_{0} k_{B} T_{c}\right)^{2}}{\left(\Phi_{0} \epsilon\right)^{3}} \approx 10^{-13} \frac{T_{c}^{2} \kappa^{4}}{\epsilon^{3}}
$$

where $B_{L L L}$ is measured in tesla. There is no clearly defined value of $\epsilon$ which will guarantee that the LLL approximation is good. Certainly, $\epsilon$ must be smaller than the value of approximately 0.3027 for which $f(\epsilon)=0$. Numerically, we find that $f(z)$ can be reasonably well approximated by a function of the form $f(z)=f_{0}(z) z^{-1 / 2}$ for $0 \leq z \leq 0.1$, where $f_{0}(z)$ is an amplitude which varies from $f(0)=\sqrt{ } \pi$ to $f(0.1)=1$, so we surmise that LLL scaling might be good for $z<\epsilon=0.1$.

Detailed measurements of the fluctuation specific heat of $\mathrm{YBaCuO}$ have recently been reported by Overendet $a l^{22}$. For this material, $T_{c}=92 \mathrm{~K}$, and we may reasonably take $\xi_{0} \approx 10 \AA$ and $\kappa \approx 100$. We then estimate the slope of the $H_{c 2}(T)$ line as $d B / d T \approx-4 \mathrm{TK}^{-1}$ and, using values of $\epsilon$ between 0.1 and 0.2 , expect that LLL scaling might set in with an applied field $B_{L L L}$ between 10 and $100 \mathrm{~T}$. In fact, the measurements of Overend et al indicate that LLL scaling fails at all fields up to $8 \mathrm{~T}$. Junod et $a l^{6}$ have recently reported measurements on the specific heat of $\mathrm{YBaCuO}$ up to 20 T. For fields greater than 2.5 T, they find that the LLL scaling form collapses their data onto a common curve, but only in a narrow range of temperature near $T_{c 2}(B)$. In the neighbourhood of the peak a little below this temperature, their data does not scale at all, in marked contrast to conventional superconductors, where the LLL scaling region extends well below this peak ${ }^{13,16}$. For this reason, we do not think that their data is really consistent with LLL scaling. There is, however, 
some indication that the scaling improves at fields of the order of 20 T. Similarly, earlier specific heat measurements by Welp et $a l^{17}$ and by Inderhees et $a l^{4}$ are at best consistent with LLL scaling only in a very narrow range of temperature.

For a conventional material with, say, $T_{c}=10 \mathrm{~K}$ and $\kappa=10$ our estimate of $B_{L L L}$ would be of the order of $10^{-4} \mathrm{~T}$. Thus, the schematic phase diagram of figure 1, together with the estimate (7.3) of $B_{L L L}$ is in reasonable accord with the observation that LLL scaling appears to work well for conventional superconductors over a wide range of fields, but seems not to work for high- $T_{c}$ materials except, perhaps, in very large fields.

Following earlier authors ${ }^{2,4}$, Overend et al attempt to fit their specific heat data to a critical-point scaling expression of the form

$$
C=C_{1}-C_{2} B^{-\alpha / 2 \nu} \mathcal{C}\left(\frac{T-T_{c}(0)}{B^{1 / 2 \nu}}\right),
$$

using the exponents of the 3-dimensional XY model, and find excellent agreement for fields up to $8 \mathrm{~T}$, apart from some rounding in very low fields, which is attributable to finite-size effects. They find, however, that equally good agreement can be obtained using the scaling variable $\left(T-T_{c 2}(B)\right) / B^{1 / 2 \nu}$ which appears in $(5.7)$, provided that the slope of the upper critical field line $-d B_{c 2} / d t$ is greater than about $5 \mathrm{TK}^{-1}$. This is consistent with the crude estimate of $4 \mathrm{TK}^{-1}$ obtained above, and with the estimate of about $7 \mathrm{TK}^{-1}$ obtained by Palstra et $a l^{23}$ by extrapolating the transport entropy of vortex motion to zero. However, it would not be consistent with the slope of about $1.8 \mathrm{TK}^{-1}$ required by Junod $e t a l^{6}$ to optimize their fits to LLL scaling.

The appearance of the scaling field $T-T_{c 2}(B)$, or of $t+e B$ in (4.2) does not seem to be an artifact of the Hartree approximation used here. It arises simply from the 
fact that the Landau eigenvalues are $k^{2}+(2 n+1) e B+t$, so that $t$ always appears in the combination $t+e B$, and this would appear to be true quite generally. Within the Hartree approximation, we see from (4.5) that the scaling variable $\theta \sim(T-$ $\left.T_{c 2}(B)\right) / B^{1 / 2 \nu}$ is itself a function of $B / \tilde{t}$ or of $B \xi_{B}^{2}$, where $\xi_{B}$ is the field-dependent coherence length, rather than the zero-field coherence length which appears in the gaussian approximation. The difference between the two variables $\left(T-T_{c}(0)\right) / B^{1 / 2 \nu}$ and $\left(T-T_{c 2}(B)\right) / B^{1 / 2 \nu}$ is proportional to $B^{(1-1 / 2 \nu)} \approx B^{0.25}$ if $\nu$ is taken to be the exponent of the 3-dimensional XY model. Since this correction varies rather slowly with $B$, the distinction between the two scaling variables is probably difficult to discern by optimizing the collapse of data onto a common curve.

\section{Conclusions}

We have used a simple Hartree approximation to investigate the critical-point and lowest-Landau-level scaling properties of high-temperature superconductors. Our principal conclusions are summarized in figure 1, which indicates that critical-point scaling is to be expected in the neighbourhood of the zero-field transition, while lowest-Landau-level scaling is restricted to a high-field region near the upper critical field line $H_{c 2}(T)$. In the critical region, the appropriate scaling variable is $\left(T-T_{c 2}(B)\right) / B^{1 / \nu}$ rather than the variable $\left(T-T_{c}(0)\right) / B^{1 / 2 \nu}$ which arises in the gaussian approximation. While the Hartree approximation does not yield accurate values for critical exponents or scaling functions, it suggests that a minimum applied field of between 10 and $100 \mathrm{~T}$ is required to observe lowest-Landau-level scaling in materials such as $\mathrm{YBaCuO}$. These conclusions appear to be consistent with current experimental observations. 
I am grateful to Alan Bray, Mark Howson, Michael Moore, Neil Overend and Myron Salamon for numerous discussions on the issues addressed in this work. 


\section{References}

${ }^{1}$ D.S. Fisher, M.P.A. Fisher and D.A. Huse, Phys. Rev. B 43, 130 (1991)

${ }^{2}$ S.E. Inderhees, M.B. Salamon, N. Goldenfeld, J.P. Rice, B.G. Pazol and D.M. Ginzberg, Phys. Rev. Lett. 60, 1178 (1988)

${ }^{3}$ S. Regan, A.J. Lowe and M.A. Howson, J. Phys: Cond. Matt. 3, 9245 (1991)

${ }^{4}$ S.E. Inderhees, M.B. Salamon, J.P. Rice and D.M. Ginzberg, Phys. Rev. Lett. 66, $232(1991)$

${ }^{5}$ M.B. Salamon, J. Shi, N Overend and M.A. Howson, Phys. Rev. B 47, 5520 (1993)

${ }^{6}$ A. Junod, E. Bonjour, R. Calemczuk, J.Y. Henry, J. Muller, G. Triscone and J.C. Vallier, Physica C 211, 304 (1993)

${ }^{7}$ B.I. Halperin, T.C. Lubensky and S-K. Ma, Phys. Rev. Lett. 32, 292 (1974)

${ }^{8}$ I.D. Lawrie, Nucl. Phys. B 200 [FS4], 1 (1982)

${ }^{9}$ I.D. Lawrie and C. Athorne, J. Phys. A 16 L587 (1983)

${ }^{10}$ C. Dasgupta and B.I. Halperin, Phys. Rev. Lett. 47, 1556 (1981)

${ }^{11}$ R.E. Prange, Phys. Rev. B 1, 2349 (1970)

${ }^{12}$ A.J. Bray, Phys. Rev. B 9, 4752 (1974)

${ }^{13}$ D.J. Thouless, Phys. Rev. Lett. 34, 946 (1975)

S.P. Farrant and C.E. Gough, Phys. Rev. Lett. 34, 943 (1975)

${ }^{14}$ G.J. Ruggieri and D.J. Thouless, J. Phys. F 6, 2063 (1976)

${ }^{15}$ N.K. Wilkin and M.A. Moore, Phys. Rev. B 47, 957 (1993)

${ }^{16}$ N.K. Wilkin and M.A. Moore, Phys. Rev. B 48, 3464 (1993)

${ }^{17}$ U. Welp, S. Fleshler, W.K. Kwok, R.A. Klemm, V.M. Vonokur, J. Downey, B. Veal and G.W. Crabtree, Phys. Rev. Lett. 67, 3180 (1991)

${ }^{18}$ S. Hikami and A. Fujita, Phys. Rev. B 41, 6379 (1990)

${ }^{19} \mathrm{~S}-\mathrm{K} . \mathrm{Ma}$, in Phase Transitions and Critical Phenomena, ed. C. Domb and M.S. 
Green, Vol. 6 p. 249 (Academic Press: London, 1976)

${ }^{20}$ S. Ullah and A.T. Dorsey, Phys. Rev. B 44, 262 (1991)

${ }^{21}$ M. Tinkham, Introduction to Superconductivity (McGraw - Hill: New York 1975)

${ }^{22}$ N. Overend, M.A. Howson and I.D. Lawrie (to be published)

${ }^{23}$ T.T.M. Palstra, B. Battlogg, L. F. Schneemeyer and J. V. Waszczak, Phys. Rev.

Lett. 64, 3090 (1990) 


\section{Figure Caption}

Figure 1. Schematic phase diagram of a high-temperature superconductor in the neighbourhood of its critical point. Shaded regions are those in which critical point or lowest-Landau-level scaling should be observed. The line $t=-e B$ corresponds to the upper critical field, while the line $\langle\phi\rangle \sim 0$ is a notional one, below which the approximation $\langle\phi\rangle \approx 0$ might be expected to fail. The curve $\tilde{t} / 2 e B=\epsilon$ represents a criterion for the validity of the lowest Landau level approximation explained in the text. 